\title{
Density Matrix Renormalization Group Study of Ultrasmall Superconducting Grains
}

\author{
J. Dukelsky ${ }^{1}$ and G. Sierra ${ }^{2}$ \\ ${ }^{1}$ Instituto de Estructura de la Materia, C.S.I.C., Madrid, Spain \\ ${ }^{2}$ Instituto de Matemáticas y Física Fundamental, C.S.I.C., Madrid, Spain
}

(Received 28 January 1999)

\begin{abstract}
We apply the density matrix renormalization group (DMRG) method to the BCS pairing Hamiltonian which describes ultrasmall superconducting grains. Our version of the DMRG uses the particle (hole) states around the Fermi level as the system block (environment). We observed a smooth logarithmiclike crossover between the few electron regime and the BCS-bulk regime.
\end{abstract}

PACS numbers: 74.20.Fg, 74.25.Ha, 74.80.Fp

The density matrix renormalization group (DMRG) [1] has been applied with great success to a large variety of systems in condensed matter and statistical mechanics (see [2] for a review). Most of these applications use the real space formulation although the DMRG can also be formulated in momentum space [3]. The starting point of the DMRG method is the breaking of the system into two pieces, the block and the environment, which are described by a finite number of states out of which one can reconstruct the ground state (GS) and the excited states of the whole system. A correct choice of the block and the environment is therefore crucial for the DMRG to work. For example, open chains are divided into left and right handed pieces linked by a couple of sites. The local interaction between the left and right pieces of the chains explains the adequacy of the DMRG in 1D problems. However, for more complicated systems, or in dimensions higher than one, there are no general rules dictating the correct DMRG breaking except the nature of the physical problem under study.

In this Letter we present a DMRG analysis of the GS of the BCS Hamiltonian used to describe the superconducting properties of ultrasmall $\mathrm{Al}$ grains, discovered by Black, Ralph, and Tinkham [4]. We show that the DMRG gives an accurate approximation to the exact GS if the block is taken to be the set of particles while the environment is taken to be the set of holes. This choice does not satisfy the local interaction rule, which is so effective in real space, for particles and holes are all coupled by the BCS Hamiltonian. Nevertheless, the projection of the GS into the particle and hole subspaces via density matrices is strongly peaked on a small number of states, which explains the applicability of the DMRG to this problem. The BCS pairing Hamiltonian used for small metallic grains is given by [5-11]

$$
\begin{aligned}
H= & \sum_{j=1, \sigma= \pm}^{\Omega}\left(\epsilon_{j}-\mu\right) c_{j, \sigma}^{\dagger} c_{j, \sigma} \\
& -\lambda d \sum_{i, j=1}^{\Omega} c_{i,+}^{\dagger} c_{i,-}^{\dagger} c_{j,-} c_{j,+},
\end{aligned}
$$

where $i, j=1,2, \ldots, \Omega$ label single particle energy levels whose energies are given for simplicity by $\epsilon_{j}=j d$, and $d$ is the average level spacing which is inversely proportional to the size of the grain. $c_{j, \sigma}$ are electron destruction operators of time reserved states $\sigma= \pm$. Finally $\mu$ is the chemical potential and $\lambda$ is the BCS coupling constant, whose appropriate value for the $\mathrm{Al}$ grains is 0.224 [8]. Given $N$ electrons they can form $P$ Cooper pairs and $b$ unpaired states such that $N=2 P+$ $b$. The Hamiltonian (1) decouples the unpaired electrons and hence $b$ is a conserved quantity. We investigate in this Letter the GS of even grains $(b=0)$ and odd grains $(b=1)$ at half filling $(N=\Omega)$ using a new version of the DMRG and compare our results with the exact Lanczos diagonalization for $N=24$ and projected BCS (PBCS) for larger $N$.

The Hamiltonian (1) has two regimes depending on the ratio $d / \Delta=2 \sinh (1 / \lambda) / N$, between the level spacing $d$ and the bulk superconducting gap $\Delta$ [5-11]. In the weak coupling region $(d / \Delta \gg 1)$, which corresponds to small grains or small coupling constants, the system is in a regime with strong pairing fluctuations above the Fermi sea which lead to logarithmic renormalizations [9]. In the strong coupling regime $(d / \Delta \ll 1)$, which corresponds to large grains or strong coupling constants, the bulkBCS wave function describes correctly the GS properties. In mean field theory the crossover between the weak and strong coupling regimes occurs at a critical value of the level spacing $d_{b}^{C}$ which is parity dependent. For even grains one has $d_{0}^{C} / \Delta=3.56$ while for odd grains $d_{1}^{C} / \Delta=0.89$ [6]. It is illustrative to consider the case where $\epsilon_{j}=0, \forall j$, so that the exact GS for $b=0$ and $N=\Omega$ is given by the PBCS state

$$
\begin{gathered}
\left|\psi_{\Omega}^{0}\right\rangle=1 /\left(\sqrt{Z_{\Omega / 2, \Omega}}\right)\left(P^{\dagger}\right)^{\Omega / 2}|0\rangle, \\
P^{\dagger}=\sum_{i=1}^{\Omega} c_{i,+}^{\dagger} c_{i,-}^{\dagger}
\end{gathered}
$$

where $|0\rangle$ is the vacuum of the electron operators and $Z_{M, N}=(M !)^{2} C_{N, M}\left[C_{N, M}=N ! / M !(N-M) !\right]$ is the norm of the state $\left(P^{\dagger}\right)^{M}|0\rangle$. Notice that (2) is the projection of the BCS state $\exp \left(P^{\dagger}\right)|0\rangle$ into the subspace with $\Omega$ electrons. On the other hand for $\lambda=0$ and $\epsilon_{j}=j d$ the GS at half filling is a Fermi sea with all the levels between $i=1$ and $i=N / 2$ occupied. This state can be 
written as

$$
\begin{gathered}
|F S\rangle=1 / \sqrt{\left(Z_{\Omega / 2, \Omega / 2}\right)}\left(B^{\dagger}\right)^{\Omega / 2}|0\rangle, \\
B^{\dagger}=\sum_{i=1}^{\Omega / 2} c_{i,+}^{\dagger} c_{i,-}^{\dagger} .
\end{gathered}
$$

Comparing (2) and (3) it is clear that the PBCS state (2) can be derived from the state (3) acting with pairs of particle-hole $(p-h)$ creation operators. With this aim we split the operator $P^{\dagger}$ as $A^{\dagger}+B^{\dagger}$, where $A^{\dagger}=$ $\sum_{i=\Omega / 2+1}^{\Omega} c_{i,+}^{\dagger} c_{i,-}^{\dagger}$. After some algebra one finds [12]

$$
\begin{gathered}
\left|\psi_{\Omega}^{0}\right\rangle=\sum_{\ell=0}^{\Omega / 2} \psi_{\ell}|\ell\rangle_{p} \otimes|\ell\rangle_{h}, \\
\psi_{\ell}=C_{\Omega / 2, \ell} / \sqrt{C_{\Omega, \Omega / 2}}, \\
|\ell\rangle_{p} \otimes|\ell\rangle_{h}=\frac{1}{Z_{t, \Omega / 2}}\left(A^{\dagger} B\right)^{\ell}|F S\rangle .
\end{gathered}
$$

Performing the $p$ - $h$ transformation $B \rightarrow B^{\dagger}$, we deduce that (2) can be written as a sum over the tensor product of particle and holes states with amplitude $\psi_{\ell}$, where $\ell$ is the associated occupation number. Tracing over the hole states one obtains from (4) a density matrix whose eigenstates are the particle states $|\ell\rangle_{p}$ with eigenvalues $w_{\ell}=\psi_{\ell}^{2}(\ell=0,1, \ldots, \Omega / 2)$. Tracing over the particle states yield identical results for the hole states. In both cases the eigenvalues of the density matrix follow the hypergeometric distribution $w_{\ell}=C_{\Omega / 2, \ell}^{2} / C_{\Omega, \Omega / 2}$, which for large values of $\Omega$ becomes a normal distribution centered at $\Omega / 4$ with quadratic deviation $\sigma=\sqrt{\Omega / 2}$. This is an interesting result because it implies that the PBCS state (2) can be approximated to a great accuracy with a number of particle and hole states of order $\sqrt{\Omega / 2}$. We expect this result to hold for generic PBCS states.

The Gaussian decay of the weights $w_{\ell}$ of the density matrix offers an ideal situation for the application of the DMRG. The DMRG works very well in the cases where the weights decay exponentially (see [13] for other types of decays). The $p$ - $h$ breaking allows for a smooth evolution of the system from a few electron regime into a superconducting one.

Before we introduce the DMRG it is convenient to perform the following canonical transformation:

$$
c_{j, \sigma}= \begin{cases}b_{\Omega / 2+1-j, \sigma}^{\dagger} & j=1, \ldots, \Omega / 2, \\ a_{j-\Omega / 2, \sigma} & j=\Omega / 2+1, \Omega,\end{cases}
$$

where the operators $a_{j, \sigma}^{\dagger}\left(b_{j, \sigma}^{\dagger}\right)$ create particles (holes) acting on the Fermi sea (3). Choosing the chemical potential $\mu$ as

$$
\mu=\frac{d}{2}(\Omega+1-\lambda)
$$

the Hamiltonian (1) has the $p$ - $h$ symmetry $a_{j, \sigma} \leftrightarrow b_{j, \sigma}$, and it can be written as

$$
H / d=K^{A}+K^{B}-\lambda\left(A^{\dagger} A+B^{\dagger} B+A B+A^{\dagger} B^{\dagger}\right),
$$

$$
K^{A}=\sum_{j=1, \sigma= \pm}^{\Omega / 2} \tilde{\boldsymbol{\epsilon}}_{j} a_{j, \sigma}^{\dagger} a_{j, \sigma}, \quad A^{\dagger}=\sum_{i=1}^{\Omega / 2} a_{i,+}^{\dagger} a_{i,-}^{\dagger},
$$

where $\tilde{\boldsymbol{\epsilon}}_{j}=j-\frac{1}{2}+\frac{\lambda}{2}$ and $K^{B}$ and $B$ can be obtained from $K^{A}$ and $A$ by the $p$ - $h$ transformation $a_{i, \sigma} \leftrightarrow b_{i, \sigma}$. We have subtracted in (7) the constant term $-\left(\frac{\Omega}{2}\right)^{2}$, which is the energy of Fermi sea $|F S\rangle$, so that the lowest eigenvalue of $H$ gives directly the condensation energy for even grains $E_{0}^{C}=\langle\psi|H| \psi\rangle-\langle F S|H| F S\rangle$. For odd grains the level located at the Fermi sea is blocked and the condensation energy can be computed following the same steps as in the even case. From now on we shall concentrate on the latter case. At half filling the GS of the Hamiltonian (7) takes the generic form

$$
|\psi\rangle=\sum_{\alpha, \beta} \psi_{\alpha, \beta}|\alpha\rangle_{p} \otimes|\beta\rangle_{h}
$$

where the particle state $|\alpha\rangle_{p}$ must have a number of particles $N_{p}$ equal to the number of holes $N_{h}$ of $|\beta\rangle_{h}$ for the GS state (9) to be nonvanishing. The DMRG is an algorithm that gives an optimal choice for the set of particle and hole states entering in (9). This set is constructed in successive steps starting from small grains. We begin with a system with $\Omega=4$ energy levels, which are chosen as the closest two particle and hole states near $\boldsymbol{\epsilon}_{F}$. This system can be represented as $\bullet \bullet \circ \circ$, where $\bullet$ stands for a particle level, while $\circ$ stands for a hole one. The Fermi energy lies in between the $\bullet$ 's and the o's. The next step is to look for the GS of the Hamiltonian (7) for $\Omega=4$ in the sector $N_{p}=N_{h}$. From the knowledge of $\psi_{\alpha, \beta}$ we define the reduced density matrix for the particle subspace

$$
\rho_{\alpha, \alpha^{\prime}}^{A}=\sum_{\beta} \psi_{\alpha, \beta}^{*} \psi_{\alpha^{\prime} \beta}
$$

The $p$ - $h$ symmetry implies that the corresponding density matrix in the hole subspace coincides with (10). The particle states which contribute the most to the GS (9) are the eigenvectors of the density matrix $\rho^{A}$ with highest eigenvalues $w_{p}[1]$. For the system $\bullet \bullet \circ \circ$ we can work with all the eigenstates of $\rho^{A}$, but in general we shall be able to keep only the $m$ most probable ones. With the information gained previously one builds the system with $\Omega=6$. The general rule is to build the system with $\Omega=$ $2(n+1)$ levels out of the system with $\Omega=2 n$. This is achieved by constructing the system with $\Omega=2(n+1)$ as a superblock of the form $\bullet \mathcal{A}_{n} \mathcal{B}_{n} \circ$, where $\mathcal{A}_{n}\left(\mathcal{B}_{n}\right)$ is the block which gives an effective description of the lowest $n$ particle (hole) levels in terms of $m$ states, while $\bullet$ and o represent the $(n+1)$ th particle and hole levels added to 
enlarge the system size. The Hamiltonian $H \bullet A B$ 。 of the superblock $\bullet \mathcal{A}_{n} \mathcal{B}_{n} \circ$ is given by

$$
\begin{gathered}
H_{\bullet A B \circ}=H_{A}+H_{B}+H_{\bullet}+H_{\circ} \\
+H_{A B}+H_{\bullet A}+H_{A \bullet}+H_{\bullet B}+H_{B \circ}+H_{\bullet \circ} \\
H_{A}=K_{n}^{A}-\lambda A_{n}^{\dagger} A_{n}, \\
H \bullet=\sum_{\sigma} \tilde{\epsilon}_{n+1} a_{n+1, \sigma}^{\dagger} a_{n+1, \sigma}-\lambda a_{n+1}^{\dagger} a_{n+1} \\
H_{A B}=-\lambda\left(A_{n} B_{n}+\text { H.c. }\right) \\
H \bullet \bullet \\
H_{\bullet A}=-\lambda\left(a_{n+1} b_{n+1}+\text { H.c. }\right) \\
H_{A}=-\lambda\left(A_{n} a_{n+1}^{\dagger}+\text { H.c. }\right)
\end{gathered}
$$

where $a_{i}^{\dagger}=a_{i,+}^{\dagger} a_{i,-}^{\dagger}$ and $A_{n}, K_{n}^{A}$ are the operators $A$ and $K^{A}$ defined in Eqs. (8) but with $\Omega / 2$ replaced by $n$. $K^{(B)}, b_{i}$, and $B_{n}$ have similar definitions. The terms $H_{B}, H_{\circ}, H_{\bullet B}$, and $H_{B}$ 。 can be derived from (12) by the $p$ - $h$ transformation $A_{n} \leftrightarrow B_{n}, a_{i} \leftrightarrow b_{i}$. The splitting (11) of the superblock Hamiltonian $H \bullet A B \circ$ recalls the one used in the momentum space DMRG [3]. However, the latter reference uses a finite system algorithm which does not exploit the $p-h$ symmetry. The DMRG provides a many body description of the blocks $\mathcal{A}_{n}$ and $\mathcal{B}_{n}$, which means that the operators acting on these blocks are represented by $m \times m$ matrices. In our case the operators that we need to keep track are $\left[A_{n}\right],\left[A_{n}^{\dagger} A_{n}\right]$, and $\left[a_{j, \sigma}^{\dagger} a_{j, \sigma}\right]$. Given these operators we can construct the superblock Hamiltonian (11) and look for the GS in the sector $N_{p}=N_{h}$ using the Lanczos method. The dimension of this Hilbert space $\left(\operatorname{dim} \mathcal{H}_{\Omega, m}\right)$ is smaller than $4 m^{2}$, for the constraint $N_{p}=$ $N_{h}$ eliminates the states away from half filling. $\operatorname{dim} \mathcal{H}_{\Omega, m}$ is usually much smaller than the exact dimension of the Hilbert space of states with $\Omega$ levels at half filling which is given by the combinatorial number $C_{\Omega, \Omega / 2}$.

Given the GS of the superblock we obtain, using Eq. (10), the density matrix of the particle system $\bullet \mathcal{A}_{n}$ and diagonalize it keeping the $m$ most probable states with weight $w_{p}$. The error of the truncation is measured by $1-P_{m}\left(P_{m}=\sum_{p=1}^{m} w_{p}\right)$. The latter states form a new basis of $\bullet \mathcal{A}_{n}$ denoted as $\mathcal{A}_{n+1}$, and they give an effective description of the particle subspace with $n+1$ levels. The hole block $\mathcal{B}_{n+1}$ is a mirror image of the particle block $\mathcal{A}_{n+1}$. The DMRG proposed above is an infinite system algorithm, which is sufficient to study moderate system sizes $(N \leq 400)$. A way to improve the numerical accuracy of the infinite system method is to choose an effective value of the coupling constant $\lambda_{n}$ (bulk) at the $n$th DMRG step in such a way that the value of the bulk gap is the one of the final system with coupling constant $\lambda=\lambda$ (bare). This is guaranteed by the equation

$$
\sinh \frac{1}{\lambda_{n}(\text { bulk })}=\frac{2(n+1)}{\Omega} \sinh \frac{1}{\lambda(\text { bare })},
$$

where $\Omega$ is the final number of levels to reach and $2(n+1)$ is the number of levels at each step. Let us now present our results. A DMRG calculation for $\Omega=24$ and $m=60$ agrees with the exact Lanczos condensation energy obtained in [10] in the first nine digits. The largest DMRG superblock matrix involved in the calculation is 3066 to be compared with the Lanczos matrix of dimension 2704156 . For $\Omega \leq 400$ and $m=60$ the condensation energy is computed with a relative error less than $10^{-4}$.

The numerical improvement achieved by the use of the effective coupling constant $\lambda_{n}$ (bulk) defined in (13) as compared with the use of $\lambda=\lambda$ (bare) in the DMRG steps is illustrated in Table I.

Let us next consider the crossover between the weak and the strong coupling regimes. The PBCS results of Ref. [11] suggest a sharp crossover between these two different regimes at characteristic level spacings $d_{0}^{C} \simeq$ $0.5 \Delta$ and $d_{1}^{C} \simeq 0.25 \Delta$. For $d<d_{b}^{C}$ the condensation energy is an extensive quantity $(\sim 1 / d)$ corresponding to a BCS-like behavior, while for $d>d_{b}^{C}$ this energy is an intensive quantity (almost independent of $d$ ) [11]. In Fig. 1 we plot the DMRG results together with those of Ref. [11]. From this comparison it is apparent that the DMRG gives significant lower energies than the PBCS ansatz. The DMRG is a variational method and in the region under study we expect our results to coincide with the exact ones with a relative error less than $10^{-4}$. Figure 1 also shows that the crossover between the BCS and the fluctuation dominated (f.d.) regimes takes place in a region which is wider than the one predicted by the PBCS approach and that there is no sign of critical level spacings $d_{b}^{C}$. A more quantitative characterization of this crossover is obtained by fitting the DMRG results to the formula (see Fig. 1)

$$
\begin{gathered}
E_{b}^{C} / \Delta=-c_{1} / \ln \left(1+c_{2} \frac{d}{\Delta}\right)+c_{3}+c_{4} \frac{d}{\Delta}, \\
c_{1}=1.48, c_{2}=3.05, c_{3}=-1.98, \\
c_{4}=0.08 \quad(b=0), \\
c_{1}=0.36, c_{2}=0.86, c_{3}=-1.95, \\
c_{4}=0.16 \quad(b=1),
\end{gathered}
$$

which interpolates between the bulk-BCS-like behavior, given by $E_{0}^{C}=-\frac{c_{1}}{c_{2}} \Delta^{2} / d(d / \Delta \ll 1)$, and the f.d. regime $(d / \Delta \gg 1)$ characterized by logarithmic

TABLE I. GS condensation energy $E_{0}^{C}$ for $\Omega=100$ and $\lambda=0.4$ computed using the effective coupling constant and the bare one. $1-P_{m}$ is the truncation error of the last iteration and $\operatorname{dim} \mathcal{H}_{100, m}$ is the largest dimension of the superblock.

\begin{tabular}{rrrcc}
\hline \hline$m$ & $E_{0}^{C}($ bare $) / d$ & $E_{0}^{C}($ bulk $) / d$ & $1-P_{m}$ & $\operatorname{dim} \mathcal{H}_{100, m}$ \\
\hline 50 & -40.44623 & -40.50014 & $2.0 \times 10^{-9}$ & 2108 \\
70 & -40.48878 & -40.50068 & $7.1 \times 10^{-11}$ & 3622 \\
90 & -40.49815 & -40.50074 & $1.1 \times 10^{-11}$ & 6306 \\
110 & -40.49983 & -40.50075 & $1.5 \times 10^{-12}$ & 9720 \\
\hline \hline
\end{tabular}




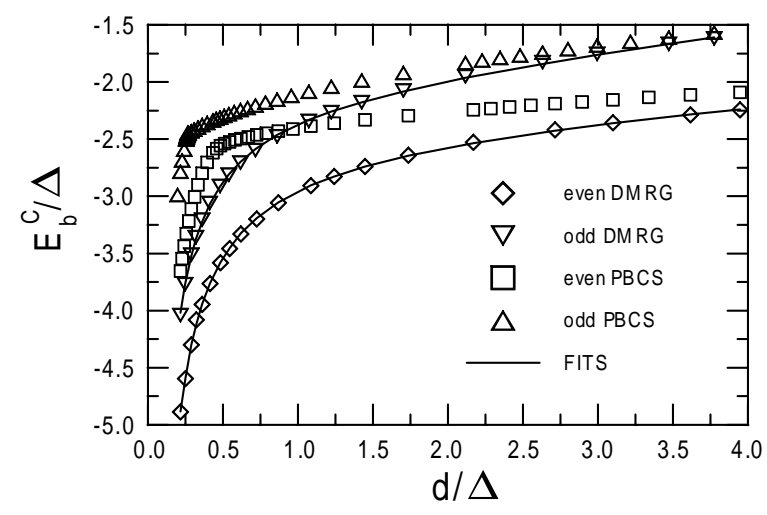

FIG. 1. GS condensation energies $E_{b}^{C}(b=0,1)$ as a function of $d / \Delta$ for $\lambda=0.224$. $\Omega$ ranges from 22 (23) up to 400 (401) for even (odd) grains and $m=60$. The PBCS results are those of Ref. [11].

corrections [9]. Indeed from (14) we get $c_{2} / c_{1}=2.06$ for $b=0$ and $c_{2} / c_{1}=2.4$ for $b=1$, which are both close to the bulk value given by 2 [8]. The previous results are consistent with the probabilities of the $m$ states kept by the DMRG as a function of the number of $p-h$ states appearing in (9), which we denote as $N_{p-h}$. Let us call $w\left(N_{p-h}\right)$ the sum of the probabilities of all the states with the same value of $N_{p-h}$. Each value of $w\left(N_{p-h}\right)$ is dominated by a single state. In Fig. 2 we plot $w\left(N_{p-h}\right)$ for some cases plotted in Fig. 1. The fast decay of these weights recalls the Gaussian decay of the eigenvalues of the density matrix of the PBCS state (2). For $\Omega=22$, $100,180,270$, and 400 the most probable states have $N_{p-h}=0,0,1,1,2$, respectively, while the next two most probable states have occupation numbers $\left|N_{p-h} \pm 1\right|$. As $\Omega$ increases the most probable state moves to higher values of $N_{p-h}$, becoming eventually commensurable with $\Omega$ in the extreme BCS regime. It may seem from these results that the $p-h$ DMRG is not capable to describe the bulk-BCS regime. This is not so because we have indeed observed this regime for higher values of $\lambda$.

In summary, we have proposed in this Letter a new version of the DMRG which is suitable to study the GS properties of the BCS pairing Hamiltonian of the ultrasmall superconducting grains. We believe that the $p-h$ DMRG formulated in this work can be applied to a more general variety of fermionic systems in condensed matter, atomic, molecular [14], and nuclear physics. Performing a Hartree-Fock transformation we can look for the best single particle basis to begin the DMRG procedure.

The main limitation concerns again the amount of important states which in general will grow with the size of the system to some power. For example, in the pairing problem it grows with the square root of the size which allows us to study large systems.

The comparison of the DMRG and PBCS results [11] shows no signs of critical level spacings separating qual-

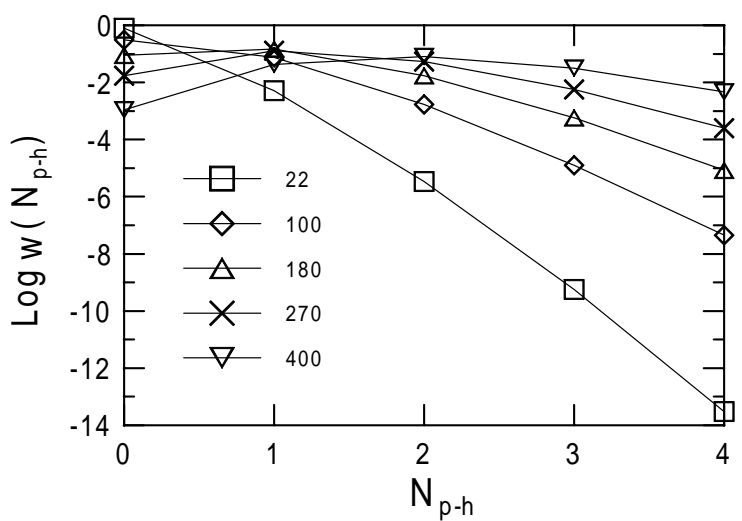

FIG. 2. DMRG weights for $m=60$ and $\lambda=0.224$ as a function of the number of $p$ - $h$ states for different values of $\Omega$.

itative different regimes. We rather observe a smooth logarithmic-like crossover which contradicts the sharp crossover predicted by the PBCS ansatz. This latter feature is an artifact of the PBCS method which is unable to capture the true nature of the crossover. In summary the fluctuations seem to play a major role for no too small grains.

We thank M. A. Martin-Delgado, S. Pittel, P. Schuck, and A. Zuker for conversations and F. Braun and J. von Delft for sending us their numerical data. This work was supported by the DGES Spanish Grants No. PB95-01123 (J. D.) and No. PB97-1190 (G. S.).

[1] S. R. White, Phys. Rev. Lett. 69, 2863 (1992); Phys. Rev. B 48, 10345 (1993); Phys. Rep. 301, 187 (1998).

[2] Density-Matrix Renormalization, Lecture Notes in Physics, edited by I. Peschel et al. (Springer-Verlag, Berlin, 1999).

[3] T. Xiang, Phys. Rev. B 53, 10445 (1996).

[4] C. T. Black, D.C. Ralph, and M. Tinkham, Phys. Rev. Lett. 76, 688 (1996); D. C. Ralph, C. T. Black, and M. Tinkham, Phys. Rev. Lett. 78, 4087 (1997).

[5] B. Jankó, A. Smith, and V. Ambegaokar, Phys. Rev. B 50, 1152 (1994); D. S. Golubev and A. D. Zaikin, Phys. Lett. A 195, 380 (1994).

[6] J. von Delft et al., Phys. Rev. Lett. 77, 3189 (1996).

[7] F. Braun et al., Phys. Rev. Lett. 79, 921 (1997).

[8] F. Braun and J. von Delft, Phys. Rev. B 59, 9527 (1999).

[9] K. A. Matveev and A. I. Larkin, Phys. Rev. Lett. 78, 3749 (1997).

[10] A. Mastellone, G. Falci, and R. Fazio, Phys. Rev. Lett. 80, 4542 (1998).

[11] F. Braun and J. von Delft, Phys. Rev. Lett. 81, 4712 (1998).

[12] P. Ring and P. Schuck, The Nuclear Many Body Problem (Springer-Verlag, Berlin, 1980).

[13] K. Okunishi, Y. Hieida, and Y. Akutsu, cond-mat/ 9810239.

[14] S. R. White and R. L. Martin, cond-mat/9808118. 